\title{
Molecular theory of hydrophobic mismatch between lipids and peptides
}

\author{
Daniel Duque, Xiao-jun Li, ${ }^{\text {a) }}$ Kirill Katsov, and M. Schick \\ Department of Physics, University of Washington, Seattle 98195-1560
}

(Received 23 January 2002; accepted 20 March 2002)

\begin{abstract}
Effects of the mismatch between the hydrophobic length $d$, of transmembrane alpha helices of integral proteins and the hydrophobic thickness, $D_{h}$, of the membranes they span are studied theoretically utilizing a microscopic model of lipids. In particular, we examine the dependence of the period of a lamellar phase on the hydrophobic length and volume fraction of a rigid, integral, peptide. We find that the period decreases when a short peptide, such that $d<D_{h}$, is inserted. More surprising, we find that the period increases when a long peptide, such that $d>D_{h}$, is inserted. The effect is due to the replacement of extensible lipid tails by rigid peptide. As the peptide length is increased, the lamellar period continues to increase, but at a slower rate, and can eventually decrease. The amount of peptide which fails to incorporate and span the membrane increases with the magnitude of the hydrophobic mismatch $\left|d-D_{h}\right|$. We explicate these behaviors which are all in accord with experiment. Predictions are made for the dependence of the tilt of a single trans-membrane alpha helix on hydrophobic mismatch and helix density. (C) 2002 American Institute of Physics. [DOI: 10.1063/1.1477927]
\end{abstract}

\section{INTRODUCTION}

The interaction of integral proteins with the lipids in which they are embedded is of great importance for membrane function. ${ }^{1}$ One principle governing this interaction is that the length of the hydrophobic segment of protein should closely match that of the membrane which it spans. ${ }^{2}$ Among the evidence that this hydrophobic matching is used in membrane organization is that the various membranes between the Golgi and the plasma membrane have different thicknesses. Proteins can be routed through the secretory pathway by increasing their hydrophobic thickness via mutagenesis and passing from one membrane to another more closely matching their new length. ${ }^{3}$ The difference between the hydrophobic length of protein and membrane, denoted hydrophobic mismatch, affects inter alia, lateral segregation of proteins in membranes, ${ }^{4,5}$ the lipid melting transition, ${ }^{6}$ and protein activity. ${ }^{7,8}$ Hydrophobic mismatch also affects the way in which the stability and the inclination of transmembrane helices change as functions of their hydrophobic length. Such information is very important in predicting transmembrane domains from potential protein sequences, ${ }^{9,10}$ a topic becoming increasingly important in biology with the completion of the Human Genome Project.

In an effort to elucidate such effects on a molecular level, Killian and co-workers ${ }^{11}$ investigated the effect of a series of hydrophobic peptides on the mean thickness of hydrated phosphatidylcholine membranes with different tail lengths. The peptides consisted of a sequence, whose length could be adjusted, of alternating leucine and alanine flanked on both sides by two tryptophans. The latter prefer to reside just below the lipid head groups, and therefore serve as anchors for the peptide. ${ }^{12}$ The $\mathrm{N}$ - and C-terminii were blocked, e.g., $\mathrm{FmAW}_{2}(\mathrm{LA})_{n} \mathrm{~W}_{2}$ Etn. The solution of peptides was di-

${ }^{a}$ Current address: The Institute for Systems Biology, 1441 North 34th St., Seattle, Washington 98103-8904. lute, a 1/30 peptide/lipid molar ratio. The results of this study which are of the most interest to us are as follows:

- peptides whose hydrophobic thickness, $d$, is smaller than that of the hydrophobic thickness, $D_{h}$, of the bilayer cause a reduction in the bilayer thickness.

- peptides whose hydrophobic thickness is larger than that of the bilayer cause an increase in the bilayer thickness. As the peptides are made longer, the membranes continue to thicken, but the increase in the membrane thickness is always less than the peptide increment.

- an increase in the magnitude of mismatch, $\left|d-D_{h}\right|$, whether due to peptides being too long or too short for the membrane, results in an increase in the fraction of peptides which fail to incorporate into the membrane. This effect has also been observed by Ren et al. ${ }^{13,14}$

The first observation is easily understood on simple energetic arguments, but the second is not. One might have expected the longer peptide simply to insert at an angle such that the membrane thickness is undisturbed.${ }^{15}$ However, the thickness is disturbed, and grows with increased peptide length, but does not track that increase identically. To understand this puzzling behavior and to isolate the various factors which bring about the net result of hydrophobic mismatch is the primary purpose of this paper.

Many theoretical approaches have been applied to the effects of mismatch. Almost all of them are phenomenological, based on treating the membrane as an elastic sheet. ${ }^{16}$ Some have, however, included in these phenomenological descriptions some properties of the lipid tails, such as the ability of the tails to tilt to accommodate the perturbation of inserted proteins. ${ }^{17}$ These approaches, which have recently been reviewed, ${ }^{18}$ are valuable in clarifying several aspects of the general problem of membrane impurities, and have even been applied ${ }^{17}$ to the lamellar-inverted hexagonal transition induced by a sufficient concentration of peptide. ${ }^{19}$ Nevertheless, they lack a direct link to the molecular details of the 
system. A major theoretical advance was the work of Fattal and Ben-Shaul ${ }^{20}$ who provided a molecular theory for the behavior of the lipid chains of the membrane. The peptides, with their hydrophobic length, were treated as providing a boundary condition on the configuration of the lipid chains. This molecular modeling was combined with phenomenological free energy contributions describing lipid headgroup repulsion and membrane solvent surface tension. In this paper we eschew phenomenological description and present a molecular theory which, from straightforward statistical mechanics, yields the free energy of the entire system, lipid and peptide. We utilize a molecular lipid model employed earlier $^{21}$ and treat the peptide, which traverses the membrane as an alpha helix, as a rigid rod. We consider a lamellar phase formed by the lipid, and investigate the effect on the period of this phase due to the addition of peptide in small concentrations. As a consequence of our calculations, we are able to reproduce all of the above-mentioned results, elucidate the reasons for the increase in membrane thickness when penetrated by long peptides, and delineate several different effects of hydrophobic mismatch.

\section{THE MODEL AND ITS SELF-CONSISTENT FIELD SOLUTION}

We consider a system of volume $V$ consisting of lipids and peptides whose numbers, $N_{l}$ and $N_{p}$, are controlled in the grand canonical ensemble by the fugacities $z_{l}$ and $z_{p}$, respectively. We take the system to be anhydrous for simplicity (see below). The lipids consist of a headgroup of volume $v_{h}$, and two equal-length tails each consisting of $N$ segments of volume $v_{t}$. Each lipid tail is characterized by a radius of gyration $R_{g}=\left(\mathrm{Na}^{2} / 6\right)^{1 / 2}$, with $a$ the statistical segment length. The peptide consists of a rigid, hydrophobic, core of $L$ segments each of length $b$ and volume $v_{c}$ and a terminal group at each end of volume $v_{e}$. We choose these end segments to be hydrophilic so that the peptide indeed models a hydrophobic segment within an otherwise hydrophilic region. The hydrophobic length of the peptide is $d=L b$.

There are four local densities which specify the state of the system. We measure them all with respect to the convenient density $v_{h}^{-1}$. They are the number density of the lipid headgroup, $v_{h}^{-1} \Phi_{h}(\mathbf{r})$, and of the lipid tail segments, $v_{h}^{-1} \Phi_{t}(\mathbf{r})$, the number density of the peptide core segments, $v_{h}^{-1} \Phi_{c}(\mathbf{r})$, and the peptide end groups, $v_{h}^{-1} \Phi_{e}(\mathbf{r})$. Note that all number densities, $\Phi(\mathbf{r})$, are dimensionless by definition.

We consider repulsive contact interactions between these four elements. In the simplest case, the strengths of the repulsive interactions between the two hydrophilic and the two hydrophobic elements are the same, $k T v_{h} \chi$, with $k$ Boltzmann's constant, and $T$ the absolute temperature. We take the total energy of interaction to be of the Flory type

$$
\begin{aligned}
E\left[\Phi_{h}, \Phi_{t}, \Phi_{e}, \Phi_{c}\right]= & k T \chi \int \frac{d \mathbf{r}}{v_{h}}\left[\Phi_{h}(\mathbf{r})+\gamma_{e} \Phi_{e}(\mathbf{r})\right] \\
& \times\left[\gamma_{t} \Phi_{t}(\mathbf{r})+\gamma_{c} \Phi_{c}(\mathbf{r})\right],
\end{aligned}
$$

where we have introduced the relative volume of the tails, $\gamma_{t}=2 N v_{t} / v_{h}$, of the peptide cores, $\gamma_{c}=L v_{c} / v_{h}$, and of the peptide end groups $\gamma_{e}=v_{e} / v_{h}$. Note that the interaction en- ergies depend upon the local volume fractions $\gamma_{e} \Phi_{e}$, etc. as opposed to the local number densities $\Phi_{e}$, etc. ${ }^{22}$

In addition to this local repulsion, we include the hardcore interactions between all particles in an approximate way by imposition of a local incompressibility constraint, i.e., that the sum of the volume fractions of all components must be unity everywhere:

$\Delta(\mathbf{r}) \equiv \Phi_{h}(\mathbf{r})+\gamma_{t} \Phi_{t}(\mathbf{r})+\gamma_{e} \Phi_{e}(\mathbf{r})+\gamma_{c} \Phi_{c}(\mathbf{r})-1=0$.

Steric, orientation-dependent interactions between the rigid peptide rods are not included. Such interactions, which would make the calculation much more difficult, can be safely ignored at the low peptide concentrations at which the experiments were carried out.

As shown earlier, ${ }^{21}$ the partition function of the system can be written in a form in which the four fluctuating densities, instead of interacting directly with one another, interact indirectly via four fluctuating fields, here denoted $W_{h}, W_{t}$, $W_{e}$, and $W_{c}$. Self-consistent field theory results when the fluctuating fields and densities are approximated by those values which extremize the free energy, $\Omega$, of the system in the presence of these fields. This free energy has the form

$$
\begin{aligned}
\frac{v_{h}}{k T V} \Omega= & -z_{l} \frac{\mathcal{Q}_{l}\left[W_{h}, W_{t}\right]}{V}-z_{p} \frac{\mathcal{Q}_{p}\left[W_{e}, W_{c}\right]}{V}+\frac{v_{h}}{k T V} E \\
& -\int \frac{d \mathbf{r}}{V}\left[W_{h}(\mathbf{r}) \Phi_{h}(\mathbf{r})+W_{t}(\mathbf{r}) \Phi_{t}(\mathbf{r})\right. \\
& \left.+W_{e}(\mathbf{r}) \Phi_{e}(\mathbf{r})+W_{c}(\mathbf{r}) \Phi_{c}(\mathbf{r})\right] \\
& -\int \frac{d \mathbf{r}}{V} \Xi(\mathbf{r}) \Delta(\mathbf{r}) .
\end{aligned}
$$

Here $\mathcal{Q}_{l}\left[W_{h}, W_{t}\right]$ is the partition function of a single lipid in external fields $W_{h}$, and $W_{t}$, and $\mathcal{Q}_{p}\left[W_{e}, W_{c}\right]$ is the partition function of a single peptide in the external fields $W_{e}$ and $W_{c}$. Note that a Lagrange multiplier $\Xi(\mathbf{r})$ has been introduced to enforce the incompressibility constraint of Eq. (2). The functions $\Xi, W_{h}, \Phi_{h}$, etc. which extremize this free energy will be denoted by their corresponding lower case letters $\xi, w_{h}$, and $\phi_{h}$. They are obtained from the five equations for the fields

$$
\begin{aligned}
& w_{h}(\mathbf{r})=\chi\left[\gamma_{t} \phi_{t}(\mathbf{r})+\gamma_{c} \phi_{c}(\mathbf{r})\right]+\xi(\mathbf{r}), \\
& w_{t}(\mathbf{r}) / \gamma_{t}=\chi\left[\phi_{h}(\mathbf{r})+\gamma_{e} \phi_{e}(\mathbf{r})\right]+\xi(\mathbf{r}), \\
& w_{e}(\mathbf{r}) / \gamma_{e}=\chi\left[\gamma_{t} \phi_{t}(\mathbf{r})+\gamma_{c} \phi_{c}(\mathbf{r})\right]+\xi(\mathbf{r}), \\
& w_{c}(\mathbf{r}) / \gamma_{c}=\chi\left[\phi_{h}(\mathbf{r})+\gamma_{e} \phi_{e}(\mathbf{r})\right]+\xi(\mathbf{r}), \\
& 1=\phi_{h}(\mathbf{r})+\gamma_{t} \phi_{t}(\mathbf{r})+\gamma_{e} \phi_{e}(\mathbf{r})+\gamma_{c} \phi_{c}(\mathbf{r}) .
\end{aligned}
$$

The field $\xi$ can be easily eliminated, while Eqs. (5) and (7) imply $w_{t}(\mathbf{r}) / \gamma_{t}=w_{c}(\mathbf{r}) / \gamma_{c}$, so that one deals essentially with three equations. The four densities are all functionals of the above fields except $\xi$ and, therefore, close the cycle of self-consistent equations:

$$
\phi_{h}(\mathbf{r})\left[w_{h}, w_{t}\right]=-z_{l} \frac{\delta \mathcal{Q}_{l}\left[w_{h}, w_{t}\right]}{\delta w_{h}(\mathbf{r})},
$$




$$
\begin{aligned}
& \phi_{t}(\mathbf{r})\left[w_{h}, w_{t}\right]=-z_{l} \frac{\delta \mathcal{Q}_{l}\left[w_{h}, w_{t}\right]}{\delta w_{t}(\mathbf{r})}, \\
& \phi_{e}(\mathbf{r})\left[w_{e}, w_{c}\right]=-z_{p} \frac{\delta \mathcal{Q}_{p}\left[w_{e}, w_{c}\right]}{\delta w_{e}(\mathbf{r})}, \\
& \phi_{c}(\mathbf{r})\left[w_{e}, w_{c}\right]=-z_{p} \frac{\delta \mathcal{Q}_{p}\left[w_{e}, w_{c}\right]}{\delta w_{c}(\mathbf{r})} .
\end{aligned}
$$

With the aid of the above equations, the self-consistent, or mean-field, free energy $\Omega_{\mathrm{mf}}$, which is the free energy function of Eq. (3) evaluated at the self-consistent field values of the densities and fields, can be put in the form

$$
\begin{aligned}
-\Omega_{\mathrm{mf}}\left(z_{l}, z_{p}, T\right)= & \frac{k T}{v_{h}}\left(z_{l} \mathcal{Q}_{l}\left[w_{h}, w_{t}\right]+z_{p} \mathcal{Q}_{p}\left[w_{e}, w_{c}\right]\right. \\
& \left.+V \xi_{0}\right)+E\left[\phi_{h}, \phi_{t}, \phi_{e}, \phi_{c}\right],
\end{aligned}
$$

where we have defined $V \xi_{0} \equiv \int \xi(\mathbf{r}) d \mathbf{r}$. All the fields, $w, \xi_{0}$, and densities, $\phi$, are functions of the fugacities, $z_{l}, z_{p}$, and temperature. All of the above-mentioned is a simple extension of previous procedure ${ }^{21}$.

There remains only to specify how the partition functions of the lipids and of the peptides are calculated. One defines the end-segment distribution function of the lipid $q(\mathbf{r}, s)$, a function of the external coordinate $\mathbf{r}$ and internal coordinate, $s$. The latter, measured along the lipid, takes the value 0 at the end of one tail, passes through $1 / 2$ at the headgroup, and takes the value 1 at the end of the other tail. Because the lipid tails are treated as completely flexible, the end-segment distribution satisfies the modified diffusion equation

$$
\begin{aligned}
& \frac{\partial q(\mathbf{r}, s)}{\partial s}-2 R_{g}^{2} \nabla^{2} q(\mathbf{r}, s)+\left[w_{h}(\mathbf{r}) \delta(s-1 / 2)\right. \\
& \left.\quad+w_{t}(\mathbf{r})\right] q(\mathbf{r}, s)=0,
\end{aligned}
$$

with initial condition

$$
q(\mathbf{r}, 0)=1
$$

From this function, one obtains the partition functions of the lipids,

$$
\mathcal{Q}_{l}=\int d \mathbf{r} q(\mathbf{r}, 1),
$$

and, from Eqs. (9) and (10), the head and tail densities

$$
\begin{aligned}
& \phi_{h}(\mathbf{r})=z_{l} \exp \left\{-w_{h}(\mathbf{r})\right\} q\left(\mathbf{r}, \frac{1}{2}-\right) q\left(\mathbf{r}, \frac{1}{2}-\right), \\
& \phi_{t}(\mathbf{r})=z_{l} \int_{0}^{1} d s q(\mathbf{r}, s) q(\mathbf{r}, 1-s) .
\end{aligned}
$$

To obtain the partition function of the peptide, one defines its end-segment distribution function $q_{p}(\mathbf{r}, \hat{\mathbf{n}}, s)$, where $\hat{\mathbf{n}}$ is a unit vector which specifies the orientation of the peptide. Because the peptide is rigid, and of length $L b$, this function satisfies the equation ${ }^{23}$

$$
\begin{aligned}
& \frac{\partial q_{p}(\mathbf{r}, \hat{\mathbf{n}}, s)}{\partial s}+L b \hat{\mathbf{n}} \cdot \nabla_{\mathbf{r}} q_{p}(\mathbf{r}, \hat{\mathbf{n}}, s) \\
& \quad+\left\{w_{e}(\mathbf{r})[\delta(s)+\delta(s-1)]+w_{c}(\mathbf{r})\right\} q_{p}(\mathbf{r}, \hat{\mathbf{n}}, s)=0,
\end{aligned}
$$

with initial condition

$$
q_{p}(\mathbf{r}, \hat{\mathbf{n}}, 0+)=\exp \left[-w_{e}(\mathbf{r})\right] .
$$

From this function, one obtains the partition function of the peptide,

$$
\mathcal{Q}_{p}=\int d \mathbf{r} \int d \hat{\mathbf{n}} q_{p}(\mathbf{r}, \hat{\mathbf{n}}, 1),
$$

and, by means of Eqs. (11) and (12), its end and core densities,

$$
\begin{aligned}
& \phi_{e}(\mathbf{r})=2 z_{p} \int d \hat{\mathbf{n}} q_{p}(\mathbf{r}, \hat{\mathbf{n}}, 1) \\
& \phi_{c}(\mathbf{r})=2 z_{p} \int_{0}^{1 / 2} d s \int d \hat{\mathbf{n}} q_{p}(\mathbf{r}, \hat{\mathbf{n}}, s) q_{p}(\mathbf{r},-\hat{\mathbf{n}}, 1-s) .
\end{aligned}
$$

To summarize: there are five self-consistent equations to be solved for the five fields $w_{h}(\mathbf{r}), w_{t}(\mathbf{r}), w_{e}(\mathbf{r}), w_{c}(\mathbf{r})$, and $\xi(\mathbf{r})$. They are Eqs. (4) to (8). The fields depend on the four densities $\phi_{h}(\mathbf{r}), \phi_{t}(\mathbf{r}), \phi_{e}(\mathbf{r})$, and $\phi_{c}(\mathbf{r})$, which depend, in turn, on these fields. The densities are given by Eqs. (17), (18), (22), and (23). Once the fields and densities are obtained, the free energy follows from Eq. (13).

We are interested in the way in which the peptides affect, on the average, a periodic array of lipid bilayers, that is, a lipid lamellar phase. We therefore assume that the lamellae are uniform in their plane, and vary only in the normal direction, specified by the coordinate $z$.

In the limit of vanishing peptide density, it is sufficient to solve for the fields and densities of the pure lipid bilayer, and then to solve Eq. (19) for the peptide end-segment distribution function in the presence of those densities. This is easily done in real space, since the solution of Eq. (19) is just

$$
\begin{aligned}
q_{p}(\mathbf{r}, \hat{\mathbf{n}}, s)= & \exp \left[-\int_{0}^{s} d t w_{e}(\mathbf{r}+t \hat{\mathbf{n}})[\delta(t)+\delta(t-1)]\right. \\
& \left.+w_{c}(\mathbf{r}+t \hat{\mathbf{n}})\right]
\end{aligned}
$$

and the fields are provided by Eqs. (6) and (7) with $\phi_{e}$ $=\phi_{c}=0$.

At nonzero peptide densities, the full set of selfconsistent equations must be solved, and it is more convenient to expand all functions of the position $\mathbf{r}$ and the director $\hat{\mathbf{n}}$ in terms of a complete set of basis functions. ${ }^{23,24}$

$$
g(\mathbf{r}, \hat{\mathbf{n}})=\sum_{l, m=0}^{\infty} g_{l, m} f_{l, m}(z, \cos \theta),
$$

where $\cos \theta$ is the projection of the unit vector $\hat{\mathbf{n}}$ onto the $z$ axis. A convenient set is

$$
f_{l, 0}(z, \cos \theta)=(2 l+1)^{1 / 2} \mathcal{P}_{l}(\cos \theta),
$$




$$
\begin{aligned}
f_{l, m}(z, \cos \theta)= & (4 l+2)^{1 / 2} \cos \left(\frac{2 \pi m z}{D}\right) \mathcal{P}_{l}(\cos \theta) \\
& \text { even } m, \\
f_{l, m}(z, \cos \theta)= & (4 l+2)^{1 / 2} \sin \left(\frac{2 \pi m z}{D}\right) \mathcal{P}_{l}(\cos \theta) \\
& \text { odd } m,
\end{aligned}
$$

where $\mathcal{P}_{l}$ is the $l$ th Legendre polynomial, and $D$ is the period of the lamellae. The latter is determined by minimization of the free energy with respect to it. Details of the procedure for solving the self-consistent equations in this basis can be found elsewhere. ${ }^{24}$ Of importance here is that the expansion into the infinite set of basis functions must be truncated to an expansion in a finite number of such functions. We have utilized 30 values of $l$ and 10 values of $m$, or a total of 300 functions. The errors in the free energy brought about by this truncation are less than $0.1 \%$. Note that the description of the peptide, which requires nonzero values of $l$, increases the number of basis functions required by a factor of 30 . Had solvent been included, the necessary number of basis functions would have increased still further. It is for this reason that we have ignored solvent. However, as the essence of the phenomenon we study lies in the interaction of the peptide with the lipid bilayer, it seems likely that the solvent plays no crucial role. The agreement between experiment and our results supports this.

The parameters we have chosen for our calculations are as follows. The lipid is characterized by the volume of the headgroup, $v_{h}=370 \AA^{3}$, and the volume of the tails, $2 N v_{t}$ $=999 \AA^{3}$. For comparison, the volume of the phosphatidylcholine headgroup is $337 \AA^{3}$ and that of two tails with seventeen carbons and one double bond each is $985 \AA^{3}{ }^{25}$ The radius of gyration of the tails was taken to be $R_{g}=12.3 \AA$ which was found to be reasonable in a previous study. ${ }^{21}$ The peptide is characterized by the volume of the end groups, which we took to be $v_{e}=555 \AA^{3}$ each, the volume of each of its core units, $v_{c}=96.2 \AA^{3}$, and the length of each core group $b$ which we took to be the length of each amino acid in an alpha helix conformation, $1.5 \AA$. For comparison, the volume of the two tryptophans and two alanines, which were only a portion of the end groups used by de Planque et al., ${ }^{11}$ is $460 \AA^{3}$, while the average of the volumes of the core alanine and leucine units is $95.5 \AA^{3}$. The interaction strength between hydrophilic and hydrophobic elements is such that $\chi \gamma_{t}=20$, a value which places the system well within the lamellar phase, but not so strongly segregated as to require an overly large number of basis functions. We have taken the number of peptide units to vary from $L=10$ to 40 .

\section{RESULTS}

We first consider the limit in which the density of peptide is vanishingly small. The volume fraction distribution of the lipid headgroups and tails in the anhydrous lipid bilayer are shown in Fig. 1 . The period $D$, in units of the radius of gyration of the lipid tails, $R_{g}$ is $D / R_{g}=2.831$. The thickness of the hydrophobic region, $D_{h}$, as defined by those points at which the volume fraction of the tails $\gamma_{t} \Phi_{t}=0.5$, is $D_{h} / D$

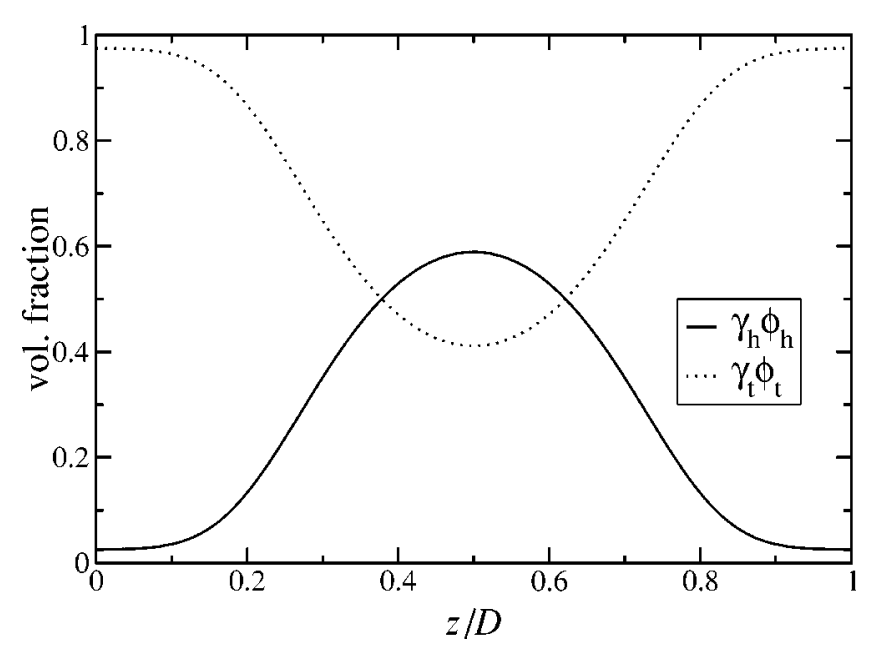

FIG. 1. Volume fraction distribution of the lipid headgroups and tails in the lamellar phase plotted vs coordinate $z$ perpendicular to the lamellae. The wavelength of the phase is $D . z / D=0.5$ corresponds to the center of the head region, and $z / D=0$ and 1 , to the center of the tail region. The interaction strength between hydrophobic and hydrophilic entities is such that $\chi \gamma_{t}=20$.

$=0.757$. The profile of Fig. 1 indicates that the system is relatively weakly segregated. Lipids in apposing bilayers have significant probability to be found in either.

It is of interest in this limit to determine whether the inserted peptide spans the bilayer and, if so, whether it inserts normal to the bilayer or at an angle, $\theta$, to it. This is readily determined. We compute the probability distribution $^{24}$

$\widetilde{P}_{l}(z, \cos \theta) \equiv q_{p}(z, \cos \theta, 1) / \int d z d(\cos \theta) q_{p}(z, \cos \theta, 1)$,

of peptide ends which are at an angle $\theta$ with the bilayer normal, when that end is located at position $z$ within the bilayer. The coordinates $z / D=0$ and 1 correspond to the center of the tail region, as in Fig. 1. The probability distribution can be calculated for peptides of different length $L b$. It is shown in Fig. 2 for an $L=20$ peptide, for which $L b / D_{h}=1.143$, that is, somewhat longer than the hydrophobic thickness of the bilayer. One sees two major and two minor peaks corresponding, respectively, to peptides which span the bilayer, and peptides which do not. We first discuss the former.

Of the two major peaks, one corresponds to a peptide inserted almost normally, at an angle such that $\cos \theta \approx-0.9$ and with one end at $z / D \approx 0.35$. This peptide would pass through the tail region at $z / D=0$. Ends of peptides in the adjacent lamellae near $z / D \approx 0.65$ are characterized by an angle $\pi-\theta$ so that the value of the cosine is the negative of that of the first peak. Thus, the second peak simply describes the other end of the rigid (and periodically repeated), peptide. As the terminal group of the peptide is anchored in the region between lipid heads and tails, it would presumably be little affected by solvent, which modifies the headgroup region.

In order to illuminate the behavior of this angle of insertion, we integrate the probability distribution of Fig. 2 over all spatial positions for a fixed angle to obtain the probability 


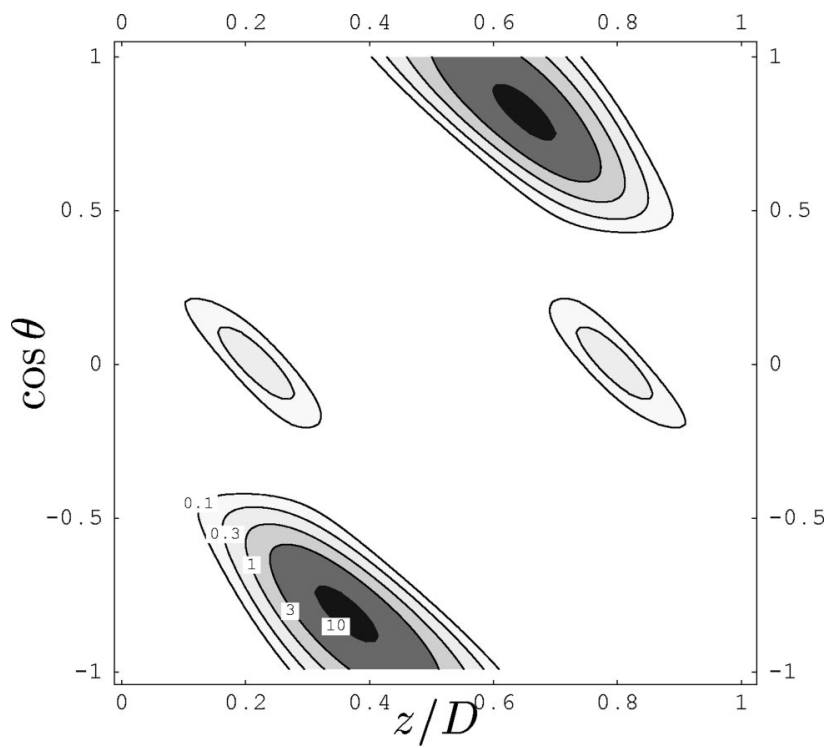

FIG. 2. Normalized probability distribution, $\widetilde{P}_{l}(z, \cos \theta)$ of the orientation, $\cos \theta$, and location, $z$, of the peptide ends for a $L=20$ peptide, of length $L b / D_{h}=1.143$, with $D_{h}$ the hydrophobic thickness of the lamellae. The center of the lipid tail region is at $z / D=0$ and 1 . Contour values are given in the legend.

distribution $P_{L}(\cos \theta)$. It is shown in Fig. 3 for several values of the hydrophobic length of the peptide $L b$ divided by the hydrophobic thickness of the bilayer, $D_{\mathrm{h}}$. From this distribution we obtain the average angle of peptide insertion and the most probable angle of insertion as a function of $L b / D_{\mathrm{h}}$. These quantities are plotted in Fig. 4 in dashed and solid lines, respectively. We observe that peptides with hydrophobic lengths $\left(L b / D_{\mathrm{h}}\right)<\left(L^{*} b / D_{\mathrm{h}}\right)=1.07$, insert normal to the bilayer. This ratio is not equal to one because our definition of the hydrophobic thickness of the membrane, $D_{\mathrm{h}}$, in terms of equal head and tail volume fractions is, like all other definitions of this quantity, a somewhat arbitrary one. Peptides

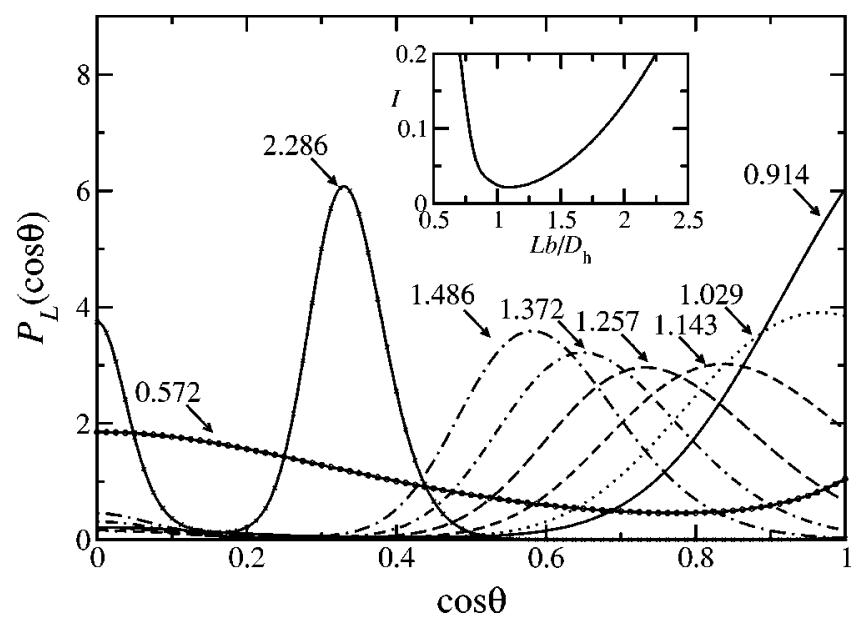

FIG. 3. Normalized probability distribution $P_{L}(\cos \theta)$ of the orientation of the peptide ends vs $\cos \theta$ for various relative peptide lengths $L b / D_{h}$, as noted on each curve. The lengths correspond to $L=16$ to $L=26$ in increments of 2, plus two extreme cases, $L=10$ and $L=40$. The inset shows the fraction, $I$, of peptides which do not insert into the bilayer as a function of $L b / D_{h}$.

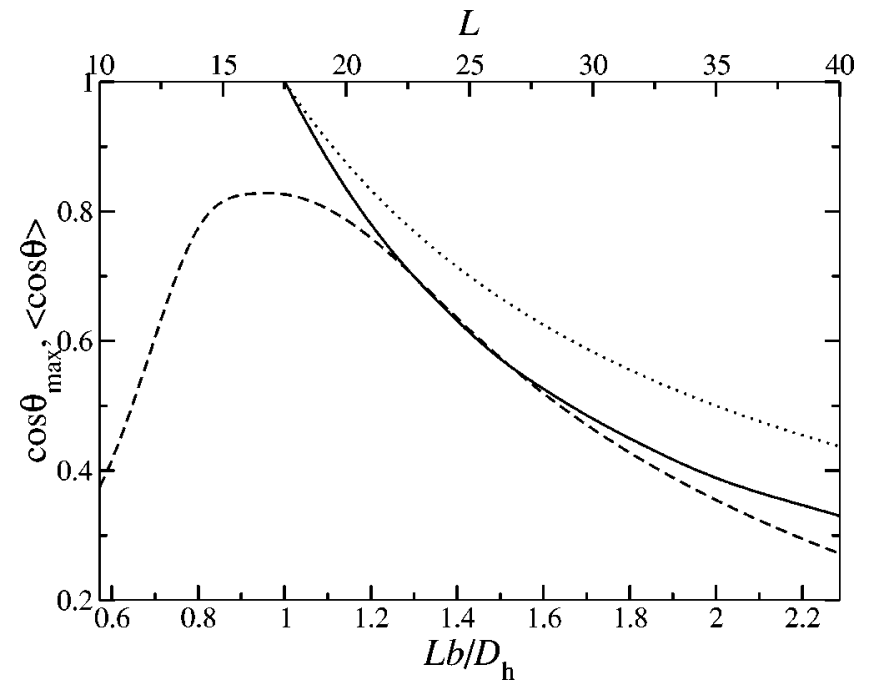

FIG. 4. Full line: location of the peak in the probability distribution function $P(\cos \theta)$ which occurs at nonzero values of $\cos \theta$; dashed line: mean value of $\cos \theta$; dotted line: simple $1 / L$ behavior. Curves are given as functions of peptide length in units of $D_{h}$ (lower $x$ scale) and $L$ (upper $x$ scale).

which are longer than $L^{*} b$ insert at a nonzero angle to the membrane normal. Their terminal groups are anchored to the interface. One might have expected that for $L \geqslant L^{*}$, the peptide would insert at an angle such that its hydrophobic thickness matched that of the membrane, i.e., $\cos \theta=L^{*} / L$. Indeed in our calculation, one sees the dependence on $L \cos \theta$ in the second term of Eq. (19). However the incompressibility condition depends only on the volume of the peptide, proportional to $L$, not on its orientation. Thus, the peptide inserts at an angle somewhat different from that which causes the hydrophobic lengths to match, and the behavior $\cos \theta=L^{*} / L$ should only be an approximation to the actual behavior. That this is indeed so is seen in Fig. 4, where we have plotted this dependence as a dotted line. One sees that our results deviate from this simple description.

Returning to Fig. 2, we also observe the two smaller peaks at $\cos \theta=0$ corresponding to a fraction of peptides which do not traverse the lamellae, but are parallel to it. As our model peptide is predominantly, but not completely, hydrophobic, the nontraversing peptides are found somewhat below the head-tail interface in the tail region of the bilayer. In order to determine how this amount of nontraversing peptides varies with the peptide length, we calculate the fraction of peptides with an end at sufficiently large angle $0 \leqslant \cos \theta$ $\leqslant 0.2$,

$$
I \equiv \int_{0}^{0.2} P_{L}(\cos \theta) d(\cos \theta) .
$$

This fraction is plotted versus $L b / D_{\mathrm{h}}$ in the inset of Fig. 3. We see that the fraction, which does not insert across the membrane, increases with the mismatch between peptide and bilayer hydrophobic thicknesses, $\left(L b / D_{\mathrm{h}}\right)-1$, irrespective of the sign of the mismatch. The largest fraction of inserted peptides occurs for $L b / D_{\mathrm{h}} \approx 1.15$.

We now consider nonzero peptide densities. We have calculated the period, $D$, of the lamellar phase as a function 


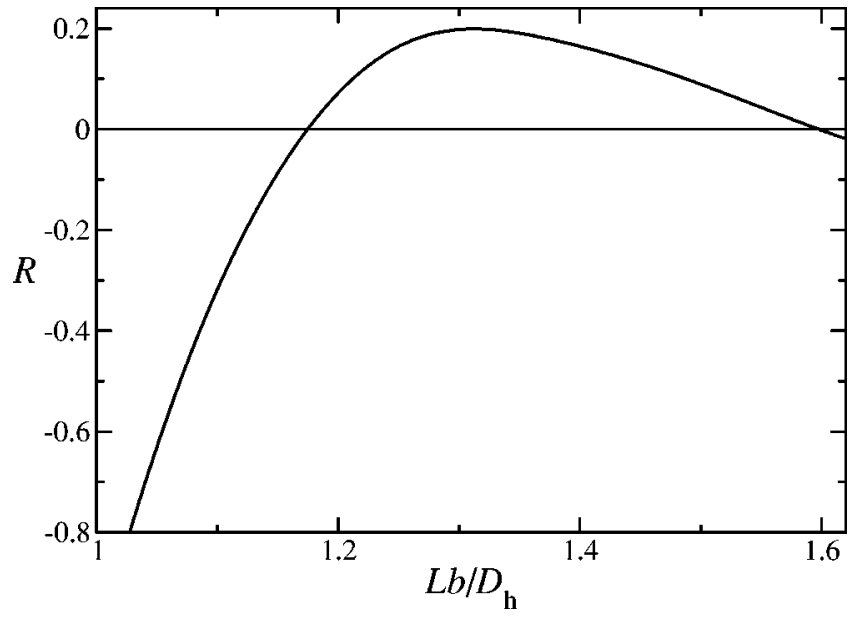

FIG. 5. Rate, $R$, of increase of relative lamellar period with volume fraction, $x_{p}$, of peptide vs relative hydrophobic length of peptide.

of peptide volume fraction, $x_{p}$, for values of $x_{p}<0.04$ and find that $D$ varies approximately linearly with it; i.e.,

$$
D\left(x_{p}\right) / D(0) \simeq 1+R x_{p},
$$

where $D(0)$ is the period of the lamellar phase in the absence of peptide. In Fig. 5 we plot the rate of bilayer thickening, $R$, versus the relative peptide thickness $L b / D_{\mathrm{h}}$. We see that the insertion of short peptides, $L b / D_{\mathrm{h}}<1.17$, causes the period, and therefore the bilayer thickness, to contract, while the insertion of long peptides causes it to increase. As the peptides become longer, the rate of increase becomes smaller. Eventually the insertion of sufficiently long peptides, $L b / D_{\mathrm{h}}>1.6$, causes the bilayer thickness to decrease.

\section{DISCUSSION}

Our results replicate and illuminate the experimental results listed in the Introduction. We recall and discuss them in turn.

The insertion of peptides whose hydrophobic thickness is less than that of the bilayer causes a reduction in the bilayer thickness.

Our calculation reproduces this result. The behavior is clearly due to simple energetic considerations.

The insertion of peptides whose hydrophobic thickness is greater than that of the bilayer causes an increase in the bilayer thickness. As the peptides are made longer by a certain amount, $\delta d$, the membranes continue to thicken, but the increase in membrane thickness is less than $\delta d$.

We reproduce these results. As noted earlier, their origin is not obvious, for energetically the peptides would be satisfied to insert at an angle such that the bilayer would not deviate from its original thickness at all. The reason for this behavior, therefore, is clearly entropic. To understand it, we need only recall the reason the bilayer thickness takes the value it does in the absence of peptide. The repulsive interaction between lipid headgroups and tails tends to crowd the head groups together which, from the constraint of incompressibility, causes the lipid tails to stretch. This tendency is opposed by the loss of tail entropy such stretching brings about. The equilibrium thickness of the bilayer results from a balance of these two tendencies. Thus in the equilibrium configuration, the lipid tails are stretched. ${ }^{20}$ Peptides, being rigid, do not stretch, and therefore lose no such entropy if the bilayer thickens, while they displace lipid tails which restrain the membrane from thickening. ${ }^{26}$ Thus it can be understood that the insertion of longer peptides causes the width of the bilayer to increase. The orientation of the peptide then adjusts to this increased width. Within this mechanism, however, there is no reason that a given increase in the length of the peptide should result in a corresponding increase in the thickness of the bilayer.

An increase in the magnitude of mismatch, whether due to peptides being too long or too short for the membrane, results in an increase in the fraction of peptides which fail to incorporate in the membrane.

Our calculation reproduces this as shown in the inset to Fig. 3. Short peptides tend not to insert for energetic reasons. Long peptides tend not to insert even though there is no energetic penalty to do so. Presumably, they fail to insert due to the loss of lipid tail entropy, which increases with the length of the hydrophobic portion of the inserted peptide.

In addition to reproducing these experimental results which demonstrates its efficacy, our model also yields predictions. As noted earlier, we found that insertion of peptides whose hydrophobic length is greater than the hydrophobic thickness of the membrane causes the membrane thickness to increase. As the peptide is made longer, the rate of increase of membrane thickness with peptide length, denoted $R$ in Eq. (27), decreases in accord with experiment. ${ }^{27}$ Strikingly, our model predicts that insertion of peptides which are very long compared to the hydrophobic thickness of the membrane ( $L b / D_{\mathrm{h}}>1.6$ in Fig. 5) actually causes the membrane thickness to decrease. We believe the reason for this is that a significant fraction of these long peptides does not traverse the membrane, but lies parallel to it. Because the peptides have a much larger end group relative to its core than do the lipids, they create relatively more free volume for the lipid tails to fill, i.e., they effectively increase the area per lipid head group. As the system is incompressible, this effect tends to make the bilayer thickness decrease. We have recently learned that this unusual thinning of the bilayer on the addition of relatively long peptides has been observed. ${ }^{28}$

Finally, our model makes predictions about the insertion of peptides at very low densities. Short peptides, whose hydrophobic thickness is less than the hydrophobic thickness of the bilayer, insert normal to it. Peptides with a hydrophobic thickness greater than that of the bilayer insert at an angle to the normal which grows with the hydrophobic mismatch. Although gramicidin channels ${ }^{29}$ are too large and complex to be described by our theory, we expect the same behavior for proteins which span the membrane with a single alpha helix. As we have ignored local effects in the plane of the membrane, the actual angle of insertion will differ somewhat from that which we have calculated, but the qualitative behavior will not be changed. Except for very large mismatches, our theory predicts the tilt to decrease with increasing peptide concentration. Although there is much data on helix tilt in specific systems, there appears to be no attempt 
at a systematic correlation of it with hydrophobic mismatch. Such data would be most interesting.

\section{ACKNOWLEDGMENTS}

The authors thank Professor J. A. Killian for useful comments and providing the Ph. D. thesis of M. R. R. de Planque. This work is supported by the NSF under Grant No. DMR9876864. One of the authors (D.D.) acknowledges support from a Spanish Ministry of Education, Culture, and Sports MEC-FPI EX 2000 grant.

${ }^{1}$ F. Dumas, M. C. Lebrun, and J. F. Tocanne, FEBS Lett. 458, 271 (1999).

${ }^{2}$ O. G. Mouritsen and M. Bloom, Biophys. J. 46, 141 (1984).

${ }^{3}$ H. R. Pelham and S. Munro, Cell 75, 603 (1993).

${ }^{4}$ D. Marsh, Mol. Membr. Biol. 12, 59 (1995).

${ }^{5}$ J. Y. A. Lehtonen and P. K. J. Kinnunen, Biophys. J. 72, 1247 (1997).

${ }^{6}$ B. Piknova, E. Perochon, and J. F. Tocanne, Eur. J. Biochem. 218, 385 (1993).

${ }^{7}$ A. Johannsson, G. A. Smith, and J. C. Metcalfe, Biochim. Biophys. Acta 641, 416 (1981).

${ }^{8}$ R. J. Froud, C. R. A. Earl, J. M. East, and A. G. Lee, Biochim. Biophys. Acta 860, 416 (1986).

${ }^{9}$ B. Rost, R. Casadio, P. Fariselli, and C. Sander, Protein Sci. 4, 521 (1995).

${ }^{10}$ J. Edelman, J. Mol. Biol. 232, 165 (1993).
${ }^{11}$ M. R. R. de Planque, D. V. Greathouse, R. E. Koeppe II, H. Schäffer, D. Marsh, and J. A. Killian, Biochemistry 37, 9333 (1998).

${ }^{12}$ J. A. Killian, I. Salemink, M. R. R. de Planque, G. Lindblom, R. E. KoeppeII, and D. V. Greathouse, Biochemistry 35, 1037 (1996).

${ }^{13}$ J. Ren, S. Lew, Z. Wang, and E. London, Biochemistry 36, 10213 (1997).

${ }^{14}$ J. Ren, S. Lew, J. Wang, and E. London, Biochemistry 38, 5905 (1999).

${ }^{15}$ J. A. Killian, Biochim. Biophys. Acta 1376, 401 (1998).

${ }^{16}$ For reviews, see Refs. 1 and 18 and T. Gil, J. H. Ipsen, O. G. Mouritsen, M. C. Sabra, M. M. Sperotto, and M. J. Zuckermann, Biochim. Biophys. Acta 1376, 245 (1998); R. M. Epand, ibid. 1376, 353 (1998).

${ }^{17}$ S. May and A. Ben-Shaul, Biophys. J. 76, 751 (1999).

${ }^{18}$ S. May, Curr. Opin. Colloid Interface Sci. 5, 244 (2000).

${ }^{19}$ P. C. A. van der Wei, T. Pott, S. Morein, D. V. Greathouse, R. E. Koeppe II, and J. A. Killian, Biochemistry 39, 3124 (2000).

${ }^{20}$ D. R. Fattal and A. Ben-Shaul, Biophys. J. 65, 1795 (1993).

${ }^{21}$ X.-J. Li and M. Schick, Biophys. J. 78, 34 (2000).

${ }^{22}$ D. R. M. Williams and G. H. Fredrickson, Macromolecules 25, 3561 (1992).

${ }^{23}$ X. Wang and M. Warner, J. Phys. A 19, 2215 (1986).

${ }^{24}$ M. W. Matsen, J. Chem. Phys. 104, 7758 (1996).

${ }^{25}$ R. Armen, O. Uitto, and S. Feller, Biophys. J. 75, 734 (1998).

${ }^{26}$ The change in rotational entropy of the peptide is negligible.

${ }^{27}$ M. R. R. de Planque, Ph. D. thesis, University of Utrecht, 2000.

${ }^{28}$ J. A. Killian (private communication).

${ }^{29}$ T. A. Harroun, W. T. Heller, T. M. Weiss, L. Yang, and H. W. Huang, Biophys. J. 76, 937 (1999). 